\title{
Estudio de la percepción sobre metodologías de enseñanza, experiencia académica previa y su relación con el rendimiento
}

\author{
Gabriela P. URREJOLA-CONTRERAS, Alejandra VÁSQUEZ-LEIVA, Caterina TISCORNIA-GONZÁLEZ, \\ Sandra LISPERGUER-SOTO, Soledad CALVO-HERRERA
}

Introducción. Se ha descrito que una favorable percepción de los estudiantes sobre un proceso de enseñanza y aprendizaje constituye un aspecto relevante que favorece el desempeño académico. Sin embargo, existe escasa información sobre otros factores de riesgo académico de los estudiantes que ingresan en la educación superior.

Objetivo. Conocer la experiencia académica previa de los estudiantes de cinesiología, su percepción sobre las metodologías de enseñanza y su relación con el rendimiento.

Sujetos y métodos. Estudio descriptivo de corte transversal, en el cual se estudiaron 108 alumnos de la Escuela de Cinesiología de la asignatura 'Anatomía del aparato locomotor'. Se diseñó y validó un cuestionario para evaluar la percepción de satisfacción y la experiencia académica previa. Posteriormente se aplicó a los estudiantes y se registraron sus calificaciones durante el semestre.

Resultados. Los estudiantes evaluaron positivamente la gestión docente en metodología y enseñanza en sus tres dimensiones, obteniendo una puntuación de 3,5 \pm 0,3. Sin embargo, el 65,7\% de los estudiantes reprobó la asignatura. Los resultados indican que no hay una relación directa entre la metodología de enseñanza y el rendimiento académico. No obstante, la experiencia académica previa parece ser de gran importancia.

Conclusión. Es necesario debatir sobre variables de índole académica, como la experiencia previa de los estudiantes, que constituyen factores de predicción del éxito académico y también aquellas que favorecen el burnout académico, e incorporar estrategias de apoyo a estudiantes que presentan estas características para un mejor desempeño académico.

Palabras clave. Educación médica. Experiencia académica. Percepción estudiantil. Rendimiento académico.

Student perception over teaching methods, academic background and its relation to academic performance

Introduction. It is known that a positive student perception about the teaching and learning process is a relevant aspect which improves academic performance. However, there are few studies that consider other academic factors acting upon students who enroll in higher education.

Aim. To find out about the academic background of physical therapy students, their perception about teaching methods and its relation to academic performance.

Subjects and methods. A descriptive cross sectional study was conducted. Sample consisted of 108 physical therapy students who enrolled in a Locomotor Anatomy course. A questionnaire to assess perceived satisfaction and academic background was designed, which was validated and then applied to students. In addition, students' grades were register during semester.

Results. Students gave positive ratings to teachers' performance about teaching methods in all three dimensions, with a score above $3.5 \pm 0.3$. Yet, $65.7 \%$ of alumni failed the course. Results showed no direct relationship between teaching methods and academic performance. However, academic background seems very important.

Conclusion. Variables such as students' academic background, which are considered predicting factors associated with academic success, should be discussed as well as those associated with academic burnout. It is recommended to incorporate supporting strategies for students with such characteristics in order to improve their academic performance.

Key words. Academic experience. Academic performance. Medical education. Student's perceptions.

Escuela de Ciencias de la Salud; Universidad Viña del Mar (G.P. Urrejola-Contreras). Escuela de Nutrición y Dietética; Facultad de Medicina; Universidad Andrés Bello; Sede Viña del Mar (A. Vásquez-Leiva). Escuela de Kinesiología; Facultad de Salud; Universidad Santo Tomás (S. Lisperguer-Soto, S. Calvo-Herrera); Viña del Mar, Chile. Escuela de Nutrición y Dietética; Facultad de Medicina; Universidad Finis Terrae; Santiago, Chile (C. Tiscornia-González).

Correspondencia:

Profa. Gabriela P. Urrejola Contreras. Escuela de Ciencias de la Salud. Universidad Viña del Mar. Salvador Vergara, 390. CP 2520000. Viña del Mar, Chile.

E-mail: gpurrejolacontreras@gmail.com

Recibido:

17.10.18.

Aceptado:

12.12.18.

Conflicto de intereses:

No declarado.

Competing interests: None declared.

(C) 2019 FEM

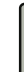

(9)

Artículo open access bajo la licencia CC BY-NC-ND (https:// creativecommons.org/licenses/ by-nc-nd/4.0/)

ISSN: 2014-9832

ISSN (ed. digital): 2014-9840 


\section{Introducción}

La actual educación en ciencias de la salud ha mostrado la necesidad de afrontar dos aspectos relevantes a la hora de mejorar el desarrollo de la docencia. El primer aspecto apunta a mejorar los elementos cualitativos de la educación como la motivación y el compromiso de los estudiantes, ya que diversos autores sugieren la existencia de una correlación positiva entre el vigor, dedicación y retención de los contenidos con la satisfacción frente a los estudios. Se ha demostrado que la satisfacción se relaciona con el éxito en el rendimiento académico [1].

El aprendizaje es un proceso dinámico y continuo que se caracteriza no sólo por utilizar la memorización de información, sino también por la capacidad del individuo de utilizar diversas habilidades cognitivas relacionadas con la evaluación de distintas situaciones, análisis de la información y su transferencia y comunicación a otros contextos, transformando esta información en nuevo conocimiento. Ello tiene indudablemente un impacto en aspectos motivacionales y en el sentido de autoeficacia que logran tener los estudiantes. Si bien estos aspectos se centran en el estudiante, los docentes deben adaptarse a la transformación y la mejora del proceso de enseñanza, donde aún predomina la entrega de contenidos en formato tradicional que dificulta la utilización de nuevas estrategias y metodologías activas en el aula. Se prioriza una formación con sólidos conocimientos teóricos, mientras que las habilidades que permiten integrar los contenidos con el resto de las asignaturas y contextualizar la docencia con la experiencia profesional sólo se han podido articular de forma parcial. Actualmente, esto ha exigido revisar el diseño de los programas universitarios y reducir las clases magistrales, dando paso a otras modalidades de aprendizaje activo e integrado [2] que permitan un aprendizaje profundo por encima de uno superficial $[3,5]$, potenciando a su vez un ambiente educacional positivo que permite generar en el estudiante una actitud activa. El ambiente educativo abarca todo lo que sucede en una institución educativa; influye en la motivación, la conducta y la adquisición de competencias, y por tanto existe una asociación entre la percepción del clima educacional y los resultados del proceso educativo. Genn [6] establece la importancia del clima educativo generado por el docente para beneficiar el aprendizaje y el rendimiento, que como expresión del aprendizaje, si bien puede analizarse desde las calificaciones, también deben considerarse otras variables, como el contexto en que estos aprendizajes se evalúan (métodos de evaluación, año de la carrera, tipo de asignatura) o la experiencia (intereses, actividades, satisfacción) y estrategias cognitivas que el alumno activa en su desarrollo [7]. De acuerdo con lo anterior, se han reconocido dos grupos de factores que influyen en el ambiente educacional: el estilo de gestión curricular, que se relaciona con la calidad de enseñanza, la claridad de los procesos, el análisis de resultados, el seguimiento de evaluaciones y los mecanismos de apoyo, y el proceso de enseñanzaaprendizaje, que comprende el currículo oculto, el entusiasmo por la carrera, su sentido y utilidad, el ambiente físico y los modelos a seguir. Estos factores impactan en la motivación y el sentido del deber que manifiesta el estudiante, lo que finalmente se correlaciona en mayor o menor medida con su proceso de aprendizaje [8].

El segundo aspecto a afrontar es la experiencia académica previa de los estudiantes. En educación médica se han propuesto y desarrollado modelos de predicción de rendimiento académico, que consideran los antecedentes curriculares del ciclo escolar, el tipo de experiencia previa al ingreso en la universidad (formación técnica o escuelas y talleres científicos, realización de asignaturas electivas en ciencias, asistencia a cursos preuniversitarios) y pruebas de selección de ingreso (prueba de aptitud académica, prueba de selección universitaria). Estas variables se combinan positivamente y se correlacionan con el éxito académico en los estudios [9].

Estos factores preparan un mejor escenario para recibir la formación de pregrado en ciencias de la salud y parecen predecir el rendimiento universitario, ya que comprende el desarrollo de hábitos de estudio, el pensamiento crítico y la adquisición temprana de estrategias de aprendizaje más eficaces [10]. En relación a esto, es importante considerar que las estrategias, los hábitos de estudio y la autorregulación constituyen una dimensión descrita en estudios previos como la combinación de recursos que utiliza el estudiante para guiar su propio aprendizaje, dentro de los cuales destacan, en términos operativos, la repetición, elaboración, organización y autorregulación metacognitiva (planificación de metas, control de la atención y regulación del aprendizaje mediante discusión y autoevaluación de lo que se pretender aprender) y la regulación/control del esfuerzo [11]. Se ha evidenciado que las bajas puntuaciones obtenidas por los alumnos en los ítems de elaboración y de organización parecen indicar que, en general, no se están consiguiendo algunos de los objetivos, como la necesidad de ayudar al estudiante a integrar los nuevos conocimientos con aquellos que ya posee (aprendizaje significativo) y desarrollar la competencia para organizar, manejar, 
relacionar y evaluar la información [11] (aprendizaje autónomo).

Cabe entonces preguntarse qué relación tiene la percepción estudiantil sobre la metodología docente y la experiencia académica previa sobre el desempeño académico de los estudiantes. Este trabajo busca establecer la relación de estas variables en el aprendizaje de la anatomía del aparato locomotor y el rendimiento académico.

\section{Sujetos y métodos}

El presente estudio es de tipo descriptivo y de corte transversal. La muestra incluyó a 191 estudiantes de cinesiología que realizaron la asignatura de 'Anatomía del aparato locomotor' durante el segundo semestre del año 2016 por primera vez en una universidad privada de la quinta región (Chile), provenientes de centros municipales de educación media, sin formación electiva o extraacadémica en ciencias. Se excluyeron estudiantes que presentaron una asistencia inferior al 75\%, ya que ello representa una menor interacción con las metodologías docentes, y con una edad superior a 25 años, debido a que estudiantes mayores ya presentaban experiencia en estudios universitarios en otras carreras o instituciones, y aquellos que estuvieran cursando la asignatura mediante procesos de intercambio con otras instituciones educativas nacionales o extranjeras. Por lo tanto, la muestra total fue de 108 estudiantes de la carrera de cinesiología de la asignatura ya mencionada, que estuvieron con diferentes docentes de cátedra (modalidad de clase teórica expositiva) y laboratorio (modalidad de clase práctica de reconocimiento de estructuras), pero bajo el mismo modelo y planificación de actividades.

Una revisión de la bibliografía permitió establecer los aspectos más relevantes para determinar la percepción de los estudiantes sobre la acción que ejercen los docentes en materia educativa, que incluye estrategias metodológicas tanto de entrega de contenidos y de evaluación, como también habilidades docentes y cualidades positivas que influyen en la adhesión de los estudiantes con la asignatura. Se formuló un cuestionario de tipo Likert, compuesto por tres criterios que aportaron los aspectos centrales de percepción y 18 indicadores relacionados con los criterios descritos: habilidades docentes, recursos metodológicos y herramientas de evaluación (Tabla). Este cuestionario fue contestado por los estudiantes según su apreciación individual de la metodología aplicada por el docente, asignándole un valor correspondiente a cada pregunta: muy bue-
Tabla. Cuestionario de percepción de la metodología docente.

\section{Criterio I: habilidades docentes ${ }^{\text {a }}$}

1. Se relacionan contenidos teóricos y prácticos haciendo uso de ejemplos reales

2. Sus explicaciones son claras, motivadoras, con adecuado ritmo para dar tiempo al aprendizaje

3. Enseña y fomenta estrategias para trabajar la asignatura y la participación e implicación de los estudiantes en cuanto a las técnicas de estudio y aprendizaje

4. El docente es responsable, competente (se sabe su materia) y prepara las clases

5. El docente dispone de buen carácter, otorga confianza y empatía a sus alumnos

6. El docente mantiene una buena comunicación, es abierto y respeta a sus estudiantes

Criterio II: recursos, materiales y métodos de entrega ${ }^{a}$

7. Utiliza medios audiovisuales (videos, PowerPoint, diapositivas, pizarra...)

8. Facilita material complementario para el aprendizaje (guías, resúmenes, esquemas...)

9. Desarrolla el laboratorio práctico basado en rotulación de modelos anatómicos, utilización de software de anatomía, actividades de body painting

10. Utiliza análisis de casos y resolución de problemas construyendo críticamente los contenidos con la participación de los estudiantes

11. Resuelve dudas en clases teóricas, practicas y tutorías

12. Clase de cátedra expositiva previa a los laboratorios prácticos para favorecer la comprensión de los contenidos

Criterio III: instrumentos de evaluación ${ }^{a}$

13. Utiliza pruebas parciales y continuas (no sólo examen final)

14. Valora trabajos y tareas prácticas (realizadas durante el taller)

15. Desarrolla pruebas formativas para que el estudiante reconozca sus errores y pueda corregirlos antes de otras evaluaciones

16. Valora no sólo la memorización, sino también el aprendizaje significativo

17. Da a conocer criterios y procedimientos de evaluación (rúbrica o pautas)

18. Las evaluaciones se adecuan a lo trabajado en la asignatura con compañeros y docentes

Experiencia académica previa ${ }^{b}$

1. ¿Ha realizado un preuniversitario en ciencias?

2. ¿Ha realizado un electivo en ciencias en la educación media?

3. ¿Presenta una prueba de selección universitaria $\geq 500$ puntos?

4. ¿Ha realizado antes esta asignatura?

a Posibilidades de respuesta: muy bueno (4 puntos), bueno (3 puntos), regular (2 puntos), malo (1 punto); b Posibilidades de respuesta: sí/no.

no (4 puntos), bueno (3 puntos), regular (2 puntos) y malo (1 punto). Se incluyó también un apartado de preguntas para determinar la experiencia académica previa de los estudiantes.

Para describir las principales características psicométricas de validez y fiabilidad, una vez construido el cuestionario se realizó una validación mediante el juicio experto de tres docentes. Los docentes escogidos reunieron los siguientes criterios: cinco años de experiencia docente en la asignatura de anatomía, cinco años de experiencia docente para la carrera de cinesiología, y formación de posgrado en docencia en educación superior.

Los datos aportados por los tres docentes retroalimentaron de forma positiva los ítems del cuestionario y las preguntas formuladas para evaluar cada 
Figura 1. Dimensiones de la percepción: habilidades docentes, recursos y materiales, e instrumentos de evaluación. Los datos representan la media y las puntuaciones mínima y máxima obtenidas en cada ítem.

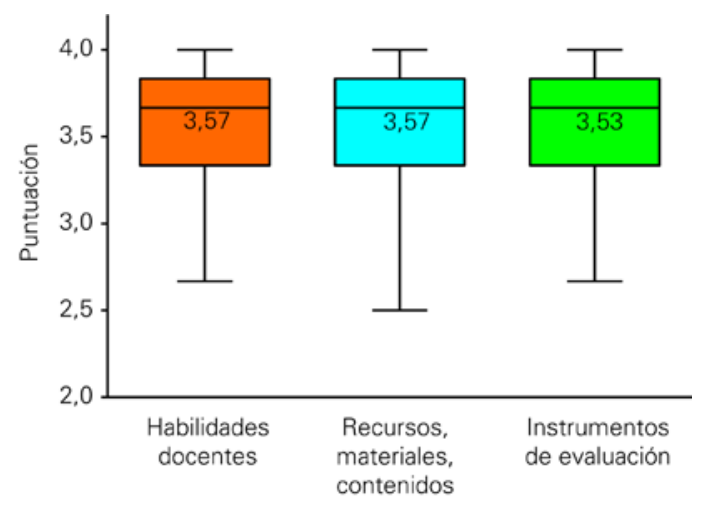

ítem; por tanto, quedó delimitada su formulación de semántica y de constructo (contenido).

Para comprobar su precisión se utilizó el programa estadístico SPSS v. 12.0, obteniendo un índice de fiabilidad para cada uno de las dimensiones que componen el cuestionario. Estos valores corresponden al índice alfa de Cronbach: habilidades docentes $(\alpha=0,73)$, recursos y materiales $(\alpha=0,70)$ e instrumentos de evaluación $(\alpha=0,67)$. Observando la distribución del cuestionario y la variabilidad de los ítems, se puede considerar como un nivel de consistencia interna aceptable. Posteriormente se aplicó el cuestionario a los estudiantes al inicio del semestre y se mantuvo la metodología docente de la asignatura en forma teórico-práctica. De modo paralelo, se recopilaron los datos obtenidos en el cuestionario y las calificaciones obtenidas de los estudiantes. Se considera aprobación una nota $\geq 4,0$ puntos. Se tabularon los datos en una plantilla Excel para su posterior análisis en el programa SPSS. En cuanto a los aspectos éticos asociados a este trabajo, se consideró la formulación de un consentimiento informado a los estudiantes y docentes participantes, que incluía: libertad de participar en la resolución del cuestionario, resguardo y protección de datos, y calificaciones y uso de la información sólo con fines académicos e investigadores.

\section{Resultados}

Globalmente, los estudiantes aprobaron la gestión de sus docentes en cada una de las preguntas apli-
Figura 2. Histograma para la distribución de notas valorado en frecuencia absoluta: el 65,7\% de los alumnos reprueba el curso.

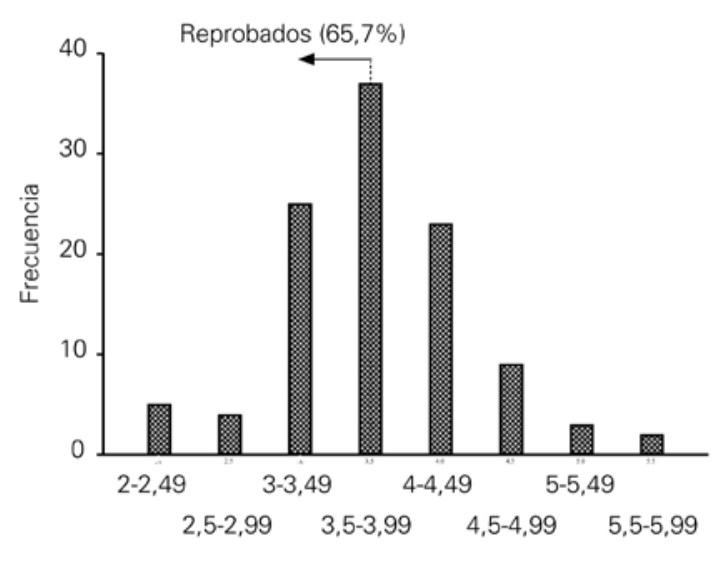

cadas en el cuestionario en relación a las tres dimensiones de la percepción: habilidades docentes, recursos y materiales, e instrumentos de evaluación. Se consideró la puntuación mínima, máxima y la media de cada una de las preguntas. En segundo lugar, sobre los resultados de las tres dimensiones del cuestionario, si bien los tres ítems obtienen una puntuación de aprobado por parte de los estudiantes, la dimensión que considera la opinión de los estudiantes sobre el diseño, frecuencia, dificultad y formato de los instrumentos de evaluación presentó una puntuación menor (Fig. 1).

En relación al rendimiento que obtienen los estudiantes en la asignatura, el porcentaje de reprobación corresponde a un $65,7 \%$. Sin embargo, tanto estudiantes aprobados como reprobados aprueban las tres dimensiones del cuestionario (Fig. 2).

Según los resultados, no se observó una correlación significativa entre la percepción de los estudiantes sobre las dimensiones del cuestionario y el rendimiento académico.

En cuanto al desglose del rendimiento, los estudiantes presentaron una mayor reprobación en la modalidad práctica (74\%), mientras que en la modalidad teórica la reprobación fue del $37 \%$.

En relación a los antecedentes curriculares y a los factores que predicen un mejor rendimiento universitario en ciencias, la mayoría de estudiantes carecen de una formación académica previa. La relación entre estos factores y el rendimiento indicó que el $83 \%$ de los estudiantes que realizaron un curso preuniversitario o electivo en ciencias durante la enseñanza media y el $100 \%$ de los estudiantes con 
una puntuación igual o superior a 500 puntos en la prueba de selección universitaria aprobaron la asignatura de 'Anatomía del aparato locomotor'. Por contra, los resultados revelan que el $100 \%$ de los estudiantes sin formación previa en ciencias y aquellos con menos de 500 puntos en la prueba de selección universitaria reprueban dicha asignatura.

\section{Discusión}

Considerando los antecedentes mencionados, las tres dimensiones sobre el quehacer docente evaluadas mediante el cuestionario revelaron que constituyen elementos básicos que favorecen el aprendizaje y la calidad educativa [12]. Si bien los estudiantes consideran que sus profesores presentan variadas habilidades en el aula, como mantener y transmitir actitudes positivas, lo que a su vez genera un clima que favorece la interacción y la entrega de los contenidos mediante variadas metodologías, el rendimiento académico tiende a ser deficiente. La apreciación de los estudiantes sobre el ítem de instrumentos de evaluación, aunque no presenta una diferencia significativa, experimentó una puntuación ligeramente inferior y puede deberse al temor que representa para ellos la instancia de someterse a una evaluación de contenidos, criterio que se ha descrito como estresor en ciencias de la salud y que repercute negativamente en el rendimiento [13].

Un segundo aspecto lo constituyen las características de riesgo que presentan los estudiantes evaluados, razón que puede explicar el bajo rendimiento obtenido. Esto implica que los estudiantes presentan dificultades para decodificar la información y utilizarla de forma efectiva [14]. Como reflexión, la muestra estudiada en este trabajo presenta este tipo de merma, ya que los estudiantes provienen de sistemas municipales de educación, un estrato socioeconómico vulnerable, y con puntuaciones bajas en las pruebas de selección universitaria de admisión. Si bien la aproximación del rendimiento es multifactorial -y este trabajo sólo consideró en el cuestionario aspectos relacionados con la experiencia académica previa de los estudiantes-, diversos autores la han considerado como el indicador más predictivo del éxito académico al reunir y conjugar en el estudiante el dominio de habilidades y conocimientos básicos, como una mejor ortografía y comprensión lectora, generación de pensamiento elemental, capacidad de análisis y síntesis, y elección de decisiones sobre cómo afrontar una evaluación. Estas capacidades facilitan la transición desde la enseñan- za media a la educación superior e impacta positivamente en el rendimiento del alumnado $[15,16]$.

Con respecto a la diferencia en el rendimiento, se observó una mayor dificultad de los estudiantes para la aprobación de la modalidad práctica (laboratorio). La mayor parte de los estudiantes reprueba la asignatura debido al bajo desempeño en la evaluación práctica de reconocimiento de muestras y figuras o modelos anatómicos. Los instrumentos de evaluación utilizados en la enseñanza de la anatomía han sido objeto de una amplia crítica. Se sabe que las condiciones socioeconómicas se vinculan con el grado de formación previa de los estudiantes y parecen ser un factor importante en el desempeño y logro de resultados en el aprendizaje de esta materia. Por otro lado, los instrumentos de evaluación de selección única, tradicionalmente utilizados en la modalidad teórica, en ocasiones representan un sesgo y se ha podido esclarecer que la evaluación práctica de la anatomía humana, en ocasiones cuestionada, ha sido capaz de nivelar y objetivar la medición de los contenidos en los estudiantes, tal como mencionan Inzunza et al [17], determinando que los resultados que obtienen los estudiantes en esta evaluación resultan ser homogéneos independientemente de su mayor o menor formación académica en el momento de ingresar en una carrera de ciencias de la salud.

En relación a las debilidades de esta investigación, una limitación es no haber considerado las variables propias de los estudiantes, como las dimensiones psicológicas y dificultades individuales que constituyen el burnout académico, lo que Martínez y Salanova [18] describen como 'obstáculos' de naturaleza académica, social-ambiental e intrapersonales del alumnado, presentes en las implicaciones negativas del rendimiento y en la deserción universitaria temprana. Como resultado, diversas investigaciones en educación de ciencias de la salud han categorizado que estas dimensiones presentes en los estudiantes demuestran niveles de agotamiento emocional, despersonalización, excesiva competitividad, falta de eficacia y sensación de incompetencia respecto a sus propias actividades académicas, lo que impacta negativamente en el rendimiento [19-21].

Una de las fortalezas de este trabajo es que permite generar un escenario de diagnóstico en relación a la percepción de las habilidades docentes, recursos y materiales e instrumentos de evaluación que se utilizan para la enseñanza y el aprendizaje. A pesar de tener una favorable percepción en estas dimensiones, el rendimiento académico es deficiente, por lo que resulta imperativo debatir, proponer e incorporar estrategias que puedan ser un apoyo di- 
recto en los estudiantes que presentan aquellos factores de riesgo en relación a su experiencia académica previa. Urge reflexionar sobre mejores estrategias de estudio que consideren eficacia y funcionalidad para afrontar asignaturas con gran volumen de contenidos para estudiantes que presenten estas carencias.

Bibliografía

1. Parra P. Relación entre el nivel de engagement y el rendimiento académico teórico/práctico. Rev Educ Cienc Salud 2010; 7: 57-63.

2. Gal-Iglesias B, De Busturia-Berrade I, Garrido-Astray MC. Nuevas metodologías docentes aplicadas al estudio de la fisiología y la anatomía: estudio comparativo con el método tradicional. Educ Med 2009; 12: 117-24.

3. Olivares M, Valencia, C, Mujica, M. Desempeño del estudiante de medicina y rendimiento académico en anatomía microscópica. Educere 2010; 48: 117-25.

4. Zúñiga D, Mena B, Oliva R, Pedrals N, Padilla O, Bitrán M. Modelos de predicción del rendimiento académico de los estudiantes de medicina en el ciclo básico y preclínico. Un estudio longitudinal. Rev Med Chile 2009; 137: 1291-300.

5. Herrera C, Pacheco J, Rosso F, Cisterna C, Aichele D, Becker S, et al. Evaluación del ambiente educacional preclínico en seis escuelas de medicina en Chile. Rev Med Chile 2010; 138: 677-84

6. Genn JM. Curriculum, environment, climate, quality and change in medical education -a unifying perspective. Med Teach 2001; 23: 337-44.

7. Navarro N, Zamora J. Evaluación del rol del tutor: comparación de percepción de estudiantes de las carreras de la salud. Investigación en Educación Médica 2017; 7: 10-7.

8. Gerardo J, Avendaño R, Martínez J. El uso de la tecnología en la enseñanza de la anatomía en México y su comparación con la enseñanza internacional. Rev Fac Med (Mex) 2014; 57: 31-9.

9. Bastías G, Villarroel L, Zúñiga P, Marshall G, Velasco N, Mena B. Desempeño académico de los estudiantes de medicina: ¿un resultado predecible? Rev Med Chile 2000; 128: 671-8.
10. Vélez A, Roa C. Factores asociados al rendimiento académico en estudiantes de medicina. Educ Med 2005; 8: 74-82.

11. Torrano F, Soria M. Una aproximación al aprendizaje autorregulado en alumnos de educación secundaria. Contextos Educativos 2016; doi: 10.18172/con.2838.

12. Tabera MV, Álvarez MJ, Hernando A, Rubio, M. Percepción de los estudiantes universitarios de ciencias de la salud sobre las actitudes de los docentes y su influencia en el clima de aprendizaje. Revista Complutense de Educación 2015; 26 : 275-93.

13. González R, Fernández R, González L, Rodríguez C. Estresores académicos percibidos por estudiantes universitarios de ciencias de la salud. Fisioterapia 2010; 32: 151-8.

14. Malander N. Estrategias de aprendizaje y hábitos de estudio en el nivel superior: diferencias según el año de cursado. Apuntes Universitarios 2014; 4: 9-22.

15. Soria K, Zúñiga $\mathrm{S}$. Aspectos determinantes del éxito académico en estudiantes universitarios. Formación Universitaria 2014 7: 41-50.

16. Garzón R, Rojas MO, Del Riesgo L, Pinzón M, Salamanca L. Factores que pueden influir en el rendimiento académico de estudiantes de bioquímica que ingresan en el programa de medicina de la Universidad del Rosario-Colombia. Educ Med 2010; 13: 85-96.

17. Inzunza O, Schneeberger D, Salgado G, Cantin M. Actividades prácticas en anatomía: factor nivelador de los contrastes de origen entre los alumnos de medicina. Int J Morphol 2017; 35: 494-9.

18. Martínez I, Salanova M. Niveles de burnout y engagement en estudiantes universitarios. Relación con el desempeño y desarrollo profesional. Revista de Educación 2003; 330: 361-84.

19. Sanguinetti J, López R, Vieta M, Berruezo S, Chagra C. Factores relacionados con el rendimiento académico en alumnos de fisiopatología. Investigación en Educación Médica 2013; 2: 177-82.

20. Caballero C, Hederich C, Palacios J. El burnout académico: delimitación del síndrome y factores asociados con su aparición. Revista Latinoamericana de Psicología 2010; 42: 131-46.

21. Glaria R, Carmona L, Pérez C, Parra P. Burnout y engagement académico en fonoaudiología. Investigación en Educación Médica 2015; 5: 17-23. 